\title{
Central line-related bloodstream infections and microbiological study in an Egyptian Ministry of Health Hospital
}

\author{
Khalil Shaaban Nahla ${ }^{1}$, Ismaeel Sayed Manal $^{2}$, Mohamed Abdnaby Gehan ${ }^{3}$
}

1. Critical Care and Emergency Nursing Department, Faculty of Nursing, Cairo University, Egypt.

2. Critical Care and Emergency Nursing Department, Faculty of Nursing, British University in Egypt.

3-Infection Control Department,Al Haram Hospital, Ministry of Hospital, Egypt.

\begin{abstract}
:
Background: Central line-associated bloodstream infections continue to be one of the most important health problems in ICU. Objectives: To identify the central line-related bloodstream infections and their microbiological characteristics Specific objectives to the identify the incidence, predisposing factors of central line-related bloodstream Infections and study the antibiotic sensitivity patterns of the isolated organisms.

Material and Methods: Patients' demographic, clinical data, central venous catheter related data,and blood culture specimen collection data were collected utilizing a descriptive exploratory design from a purposive sample of 120 ICU patients underwent insertion central venous catheter.

Results: out of 120 central line catheters sent for culture from patients immediately and seven days after insertion., 13(10.83\%) were positive seven days after insertion. Of the 13 infection cases, 10 cases were Staphylococcus epidermis and the others were Staphylococcus aureus. In addition, most infected patients were overweight and obese, diabetic, had sub-clavian route, and their length of hospital stayed more than7 days.

Conclusion: $10.83 \%$ developed bacterial blood stream infection 7 days after insertion of central venous catheters, and most of bacterial isolates were Staphylococcus epidermis and were sensitive to Vancomycin and Amikain.

Keywords: Bacterial Infections, Bacterial Isolates, Antibiotic Resistance.

DOI: https://dx.doi.org/10.4314/ahs.v20i1.21

Cite as: Nabla KS, Manal IS, Gehan MA. Central line-related bloodstream infections and microbiological study in an Egyptian Ministry of Health Hospital. Afri Health Sci. 2020;20(1):158-67. https:/ / dx.doi.org/10.4314/abs.v20i1.21
\end{abstract}

\section{Introduction}

Central venous catheters (CVCs) play a vital role in infusing medications, withdrawing venous samples and strict monitoring in critically ill patients. ${ }^{1}$ Utilization of CVCs has been concerned with various risks and complications. The most common risk is catheter-related blood-stream infections (CRBSIs) ${ }^{2}$. Several identified risk factors of CRBSI have been categorized into catheter-related factors, patient-related factors or technical related factors. ${ }^{3}$ Critical care nurses play an important role in managing their related factors by application of strict aseptic tech-
Corresponding author:
Khalil Shaaban Nahla,
Critical Care Nursing, Faculty of Nursing,
Cairo University.
Tel: +201066225938
Email: nahlakhali128@yahoo.com

niques and compliance with evidence-based guidelines that advised by the Centers for Disease Control and Prevention. ${ }^{4,5}$

Gram-positive cocci followed by Gram-negative bacilli are responsible for a higher proportion of catheter-related infections in the intensive care unit (ICU) than other clinical settings. ${ }^{6}$ Forming of biofilm in device-related infection has not only been reported as a significant factor in a catheter-related infection but also include resistance to antimicrobial therapy. ${ }^{7}$ The preventive measures like the utilization of antimicrobial-impregnated catheters and utilization of strict aseptic technique during catheter insertion using maximal sterile barrier (MSB) precautions have been proven to decrease the rate of bloodstream infection. ${ }^{4}$ With resulting lowered health care costs. ${ }^{8}$ Due to few data of central venous catheter bloodstream infection (CVC-BSI) from health care settings in our country, this study was conducted in one ICU of the 
ministry of health hospital determining the incidence, risk factors, microbes isolates and investigating antimicrobial susceptibility and resistance by CVC-BSI isolates.

\section{Research questions:}

To fulfill the main objective of this study, the following research questions were stated:

1 -what is the incidence rate of CLABSI among the studied ICU patients?

2- What are the predisposing factors to CLABSI among the studied ICU patients?

3-What are the microorganisms responsible for incidence of to CLABSI among the studied ICU patients?

4- What are the antibiotic sensitivity patterns of the isolated organisms among the studied ICU patients?

\section{Subjects and methods}

Design: a descriptive exploratory design was adopted. Consecutive critically ill patients were engaged into the study between September 2015 and September 2016

\section{Setting}

The study was conducted at a critical care units, at a ministry of health hospital in Giza, Egypt . That hospital manages 5000 cases / year. The nurse patient proportion is $1: 3$

\section{Sample}

A purposive sample consisting of 120 patients admitted to the critical care unit and was subjected to insertion of central venous catheters at one of Ministry of Health Hospitals. The inclusion criteria included adult patients over 18 years who had no blood stream infection before insertion of central venous catheters. Blood cultures were withdrawn immediately and seven days after insertion of central venous catheter

\section{Methods}

\section{The following tools were utilized for data collection}

-Demographic and patients' clinical data: They included data such as age, sex, body mass index, , pre-morbid disease, length of hospital stay.

-Central venous catheter related data: It included site of insertion, number of catheter lumen, use of guide wire, duration of insertion, duration of Cather in situ, and location of insertion

-Blood culture Specimen collection: This Procedure was performed by ICU nurses. It included hand washing be- fore the procedure, antisepsis of the puncture local area with $10 \%$ povidone and wearing the sterile gloves. Later, Blood samples that were collected under aseptic precautions from the peripheral vein for qualitative culture were incubated at $37^{\circ} \mathrm{C}$. Sub-cultures were made on MacConkey's and blood agar plates after 24, 48 and $72 \mathrm{hr}$ and incubated for $24 \mathrm{hrs}$ at $37^{\circ} \mathrm{C}$. The organisms were identified by colony morphology, gram staining, and biochemical tests performed by routine laboratory techniques ${ }^{9}$

\section{Antibiotic sensitivity testing:}

Antibiotic sensitivity test of bacterial isolates on $23 \mathrm{com}-$ monly used antibiotics were done on MullerHinton agar medium by disk diffusion technique according to National Committee for Clinical Laboratory Standards (NCCLS) as indicated by ${ }^{10}$ utilizing Disc of antibiotics such as Erythromycin $(15 \mu \mathrm{g})$, Cephalexin $(30 \mu \mathrm{g})$, Ceftriax-

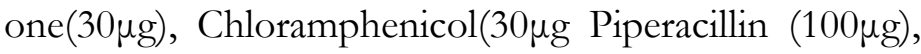

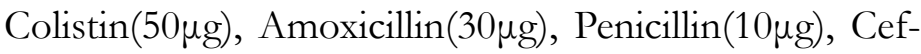
tazidime $(30 \mu \mathrm{g})$, Cloxacillin $(1 \mu \mathrm{g})$, Nalidixic acid(30 $\mu \mathrm{g})$, Ciprofloxacin $(5 \mu \mathrm{g})$, Cotrimoxazole $(25 \mu \mathrm{g})$ Levoflox-

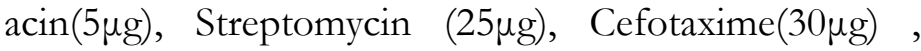

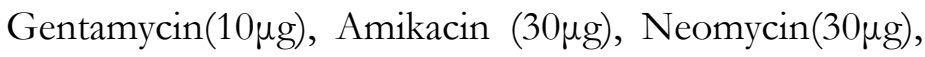
Vancomycin(30 $\mu \mathrm{g})$, Azithromycin $(30 \mu \mathrm{g})$,$) , Tetracycline$ $(30 \mu \mathrm{g})$, Imipenem $(10 \mu \mathrm{g})$ and were put on each isolate cultures respectively and incubated at $37^{\circ} \mathrm{C}$. Later, after 24 hours of incubation, the diameter of zone of inhibition that surrounds the antibiotics was calculated as recommended by Clinical Laboratory Standard Institute. ${ }^{11}$

\section{The procedure of data collection}

Once the patients' inclusion criteria were met, the patients were contacted and interviewed by the researchers to explain the procedural steps of the CVP insertion and withdrawing sample of blood culture. Later the written consents were obtained from the patients to participate in the study. Next, the patients' demographic data and clinical profile were completed by the researchers from the medical records including age, sex, body mass index, pre-morbid disease, length of hospital stay. This phase lasted 30 minutes for each patient. researchers attended the Later , the nurse researchers attended the procedure of insertion of Central venous catheters for each patient. Central venous CVCs were supplied as an available kit and inserted using a Seldinger technique by intensive care physician. Insertion was carried out under strict aseptic techniques (gowns, gloves, and masks). The insertion site ( jugular or subclavian ) was cleaned with $10 \%$ povidone-iodine 
solution for atleast 1-2 min and a sterile field ensured with long drapes. After sterilization of the insertion site, the physician underwent the insertion process. During this invasive intervention, the researchers observed the procedural steps and recorded the CVCs related data including the site of insertion, duration of insertion, the use of Guidewire, the number of lumens, and duration of Cather in situ

After immediate completion of CVC insertion, the experienced and trained nurses withdrew the venous blood culture. The site of insertion was covered by sterile gauze and a transparent adhesive tape was applied. This phase took 30-45 minutes for each patient. Later, collected samples were aseptically transferred to the laboratory to be cultured. Dressings were changed if the gauze became soiled. CVCs were not routinely replaced unless they were suspected of being infected. The blood culture was withdrawn again on the seventh day after insertion. After 72 hours, the researcher got the patients' blood culture reports and recorded them. In the case of positive blood culture, the bacterial isolates and sensitivity test was carried out and recorded. The overall data collection took 16 months starting from May 2015 to September 2016.

\section{Ethical consideration}

This study was approved by the ethics committee of faculty of nursing, Cairo University and head of ICU at $\mathrm{Al}$ Haram Hospital in Egypt. Each patient / relative was contacted to explain the nature of the study. A written informed consent was obtained from all participants before engagement in the study. Anonymity and Confidentiality of patients' data were secured throughout the study.

\section{Content validity and reliability}

Content validity of the researchers' developed tools such as observational checklist of central venous catheter related data and patients' clinical profile were reviewed by a panel of nine health care experts in the fields of critical care nursing and bacteriology. Based on experts' opinions, the authors calculated the content validity in- dex (CVI) to investigate the quantitative content validity of the established data collection tools. For calculating content validity index in our study, we sought the elected professionals to investigate, clarity, relevancy and simplicity of each element by using a 4-point Likert scale. ${ }^{12}$ The critical value point for CVI was accepted at $0.79 .{ }^{13}$ Items having a CVI of 0.79 or higher were included in the final form of observational checklist and patient' clinical profile. Concerning content validity ratio (CVR,) the experts scored each item on a 3-point Likert scale-Essential (scored 1), Useful but not essential (scored 2) and not essential (scored 3). In respect to the number of experts and the critical values of CVR as proposed by. ${ }^{13}$ We chose elements having a CVR of 0.5 or more. Reliability for the both checklist of central venous catheter related data and patients' clinical profile were determined using Cronbach Alpha $(r=0.83)$ method in a pilot study of 12 patients

\section{Data analysis}

Statistical analysis was carried out utilizing the statistical package for social sciences (SPSS) version 21. Continuous data was shown in the form of mean and standard deviation (SD), while the categorical and nominal were illustrated as frequencies and percentages. Chi-square analysis was utilized for correlates qualitative data and a value of $\mathrm{p}<0.005$ was considered significant.

\section{Results:}

\section{Demographic and clinical characteristics of the studied subjects:}

The study consisted of 120 participants in which 69 $(57.5 \%)$ were male, $51(42.5 \%)$ were female; age range with the mean age 52.2 and nearly half of them were overweight $52(43.3 \%)$.Regarding the clinical characteristics' of participants, most of them were hypertensive $82(68.3 \%)$. Moreover, the reason for insertion of CVC in most of patients was emergent intervention 108(90\%) .Concerning duration of hospital stay, two thirds of patients $81(67.5 \%)$, their duration of hospital stay was less than 10 days with the mean 9 days. (Table 1 ). 
Table 1: Demographic and Clinical characteristics of patients

\begin{tabular}{|c|c|c|c|}
\hline Variables & $\begin{array}{r}\text { Overall Number of patients } \\
120(100 \%)\end{array}$ & $\begin{array}{c}\text { Number of Catheter related blood } \\
\text { stream infections cases } \\
13(10.8 \%)\end{array}$ & P value \\
\hline \multicolumn{4}{|l|}{ Gender } \\
\hline Male & $69(57.5 \%)$ & $8(61.5 \%)$ & 0,00 \\
\hline Female & $51(42.5 \%)$ & $5(38.5 \%)$ & 0.00 \\
\hline Mean age in years & Mean $=52.2$ & $\begin{array}{ll}60 & \mathrm{SD}=15.5\end{array}$ & 0.55 \\
\hline \multicolumn{4}{|l|}{$\begin{array}{r}\text { Duration of hospital } \\
\text { stay }\end{array}$} \\
\hline$<10$ days & $81(67.5 \%)$ & $0(0 \%)$ & 0.00 \\
\hline$>10$ days & $39(32.5 \%)$ & $13(100 \%)$ & 0.00 \\
\hline Mean hospital stay & 9 days $\mathrm{S} \mathrm{D}=6.6$ & 10 days $\quad \mathrm{S} \mathrm{D}=4.6$ & \\
\hline \multicolumn{4}{|l|}{ Pre-morbidities } \\
\hline Diabetes & $38(31.7 \%)$ & $\begin{array}{ll}7 & (53.8 \%)\end{array}$ & 0.00 \\
\hline Hypertension & $82(68.3 \%)$ & $\begin{array}{ll}6 & (46.2 \%)\end{array}$ & 0.00 \\
\hline \multicolumn{4}{|l|}{ Reason for Indications } \\
\hline Emergent indication & $108(90 \%)$ & $12(92.3 \%)$ & 0.00 \\
\hline Routine replacement & $12(10 \%)$ & $1(7.7 \%)$ & 0.00 \\
\hline \multicolumn{4}{|l|}{ Body mass index } \\
\hline Under weight & $4(3.3 \%)$ & $0(0 \%)$ & \multirow{4}{*}{0.053} \\
\hline Normal weight & $33(27.5 \%)$ & $4(30.7 \%)$ & \\
\hline Over weight & $52(43.3 \%)$ & $6(46.1 \%)$ & \\
\hline Obese & $31(25.8 \%)$ & $3(23.07 \%)$ & \\
\hline
\end{tabular}

$\mathrm{P}$ value by Chi-Square test. Statistically significant

Microbiological characteristics of the studied cases Of the 120 patients enrolled in this study, all subjects' displayed negative blood culture i.e no growth of microorganisms immediately after the insertion of central venous catheters. On the other hand, $13(10.8 \%)$ displayed positive culture 7 days after insertion (fig.1). Concerning the finding pertinent to bacterial separates from blood cultures of infectious cases, it revealed that 10 cases were infected with Staphylococcus epidermis and the other 3 cases were infected with Staphylococcus aureus .(table 2).

Table 2 Bacterial isolates from blood cultures seven days after CVC insertion

\begin{tabular}{|c|c|c|c|}
\hline \multirow{2}{*}{ Variable } & \multirow{2}{*}{ Values } & \multicolumn{2}{|c|}{$\begin{array}{r}\text { Number of positive } \\
\text { Cultures } N=13\end{array}$} \\
\hline & & NO. & $\%$ \\
\hline \multirow{2}{*}{ pathogen } & Staphylococcus Epidermidis & 10 & 76.93 \\
\hline & Staphylococcus Aureus & 3 & 23.07 \\
\hline
\end{tabular}


Catheter profile and risk factors to CRBSI among the studied patients

Of the 120 patients enrolled in this study, all patients' central venous catheters were inserted in ICU setting and had triple lumen.. Regarding duration of central venous catheter insertion, all 13 CRBSI cases experienced more than 15 minutes utilizing guide wire during insertion. Moreover, more than half of patients' central venous catheters were inserted via jugular vein 63 (52.5\%). However, incidence of CRBSI was higher in patients with the sub clavian route $8(6.66 \%)$ when compared to Jugular route $5(4.16 \%)$. Regarding the duration of the catheter in place, more than two-thirds of the overall population $(67.5 \%)$ experienced less than 7 days. However, all the CRBSI cases $(10.83 \%)$ experienced more than seven days as shown in table 3 .

Table 3: Catheter related factors for the studied patients

\begin{tabular}{|c|c|c|c|}
\hline Variables & $\begin{array}{r}\text { Overall Number of patients } \\
120(100 \%)\end{array}$ & 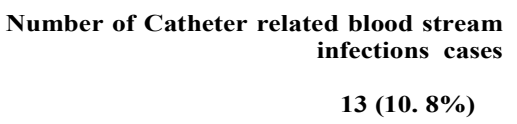 & $P$ value \\
\hline \multicolumn{4}{|l|}{ Insertion site } \\
\hline Jugular & $63(52.5 \%)$ & $5(38.46 \%)$ & 0.00 \\
\hline Sub-clavian & $57(47.5 \%)$ & $8(61.54 \%)$ & 0,00 \\
\hline \multicolumn{4}{|l|}{ Use of guide wire } \\
\hline Yes & $113(94 \%)$ & $13(100 \%)$ & 0,00 \\
\hline No & $7(6 \%)$ & $0(0 \%)$ & 0,00 \\
\hline \multicolumn{4}{|l|}{ Number of lumens } \\
\hline Single & $0(0 \%)$ & $0(0 \%)$ & \multirow[b]{2}{*}{0.00} \\
\hline Triple & $120(100 \% 0$ & $13(100 \%)$ & \\
\hline \multicolumn{4}{|l|}{ Duration of catheter in place } \\
\hline$<7$ days & $81(67.5 \%)$ & $0(0 \%)$ & 0.00 \\
\hline$>7$ days & $39(32.5 \%)$ & $13(100 \%)$ & 0.00 \\
\hline \multicolumn{4}{|l|}{ Duration of insertion } \\
\hline$<15$ minutes & $13(10.83 \%)$ & $0(0 \%)$ & \multirow[b]{2}{*}{0.00} \\
\hline$>15$ minutes & $107(89.17 \%)$ & $13(100 \%)$ & \\
\hline
\end{tabular}

$\mathrm{P}$ value by Chi-Square test. Statistically significant

Cultural sensitivity of bacterial isolates to antibiotics Regarding studying the cultural sensitivity of bacterial isolates to antibiotics in the current study, it showed that all isolates were sensitive to Vancomycin, Amikain, and
Levofloxacin. Eighty percent of bloodstream infection samples were resistant to amoxycillin clavulanic acid, $60 \%$ meropenem, 50\%, clindamycin and ceftriaxone, $40 \%$ gentamicin, penicillin and ofloxacine and 30\% imipenem.(see figure 2). 


\section{BLOOD CULTURE 2-AFTER INSERTION OF C.V.C}

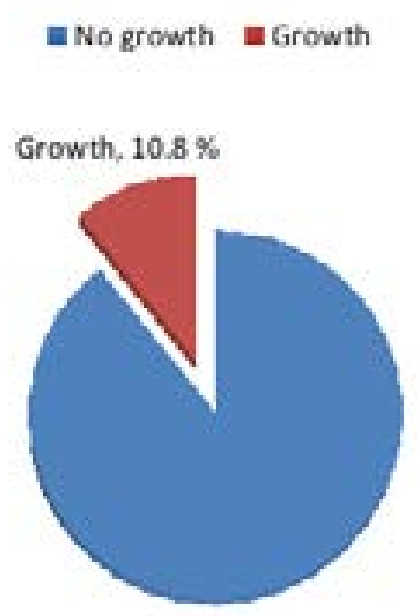

No growth, $89.2 \%$

Figure 1: Percentage distribution of patients' blood culture after CVC insertion of $(\mathrm{N}=120)$

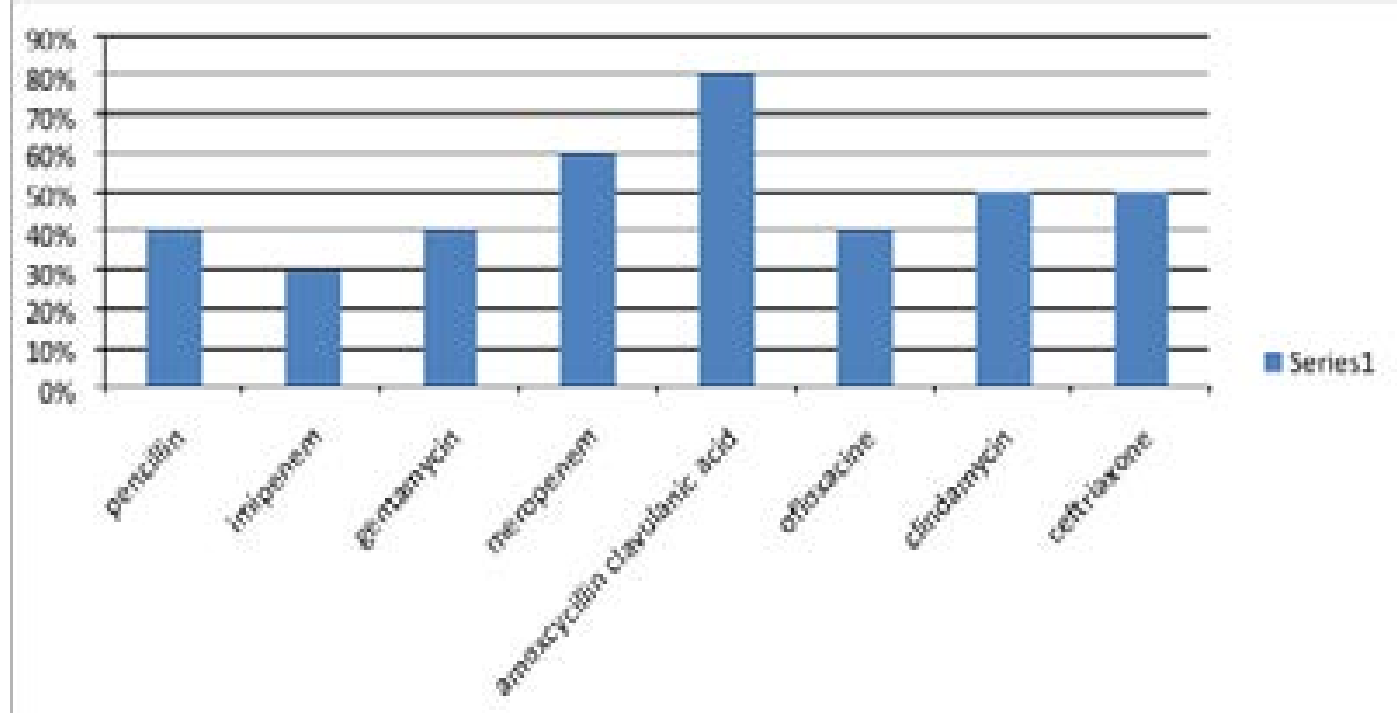

Figure 2: Antibiotic resistance of $S$. epidermidis isolates and S.aureus

\section{Discussion}

This study studied the incidence, clinical and microbiological characteristics, and risk factors for the development of catheter-related infections in a ministry of health hospital in Egypt. Of the 120 patients enrolled in this study, ${ }^{14}(10.8 \%)$ developed bloodstream infection seven days after CVC insertion (fig.1). These findings agreed with Culshaw et a ${ }^{15}$ who examined health care-associated bloodstream infections in critically ill patients and found that CLBSI accounted for $6.0 \%$. As well, our findings are consistent with ${ }^{16}$ who reported the blood infection stream rate was 10.2 per 1,000 catheters/ day. Similarly, ${ }^{17}$ revealed the bloodstream infection rate 1.4 per 1000 catheter-day.

As well, our findings are in line with previous studies done by ${ }^{18-20}$ who reported a bloodstream infection rate was 9,2 per 1,000 catheters/ day in developing countries 
that have similar difficulties in our country such as low adherence with hand wash and the nurse-to-patient staffing ratios in hospitals are low.

The detected bacterial isolates revealed that ten cases of them were infected with Staphylococcus epidermidis. The possible explanation for that finding has been attributed to inadequate compliance with the skin cleaning with antiseptics around the catheter insertion site. So, Staphylococcus epidermidis is a Gram- negative bacterium normal flora that lives on the surface of the skin and could enter the insertion site easily. This finding was consistent with ${ }^{21}$ who conducted a study about infection related processes during hemodialysis in general hospital and found that most of of bloodstream infection cases were mainly due to Staphylococcus epidermidis.

As well, our finding is agreed with a recent similar study done by ${ }^{22}$ who studied central venous catheter colonization and blood stream infection in a tertiary teaching hospital in India and revealed that negative staphylococci was the most common organism found causing central related blood stream infection. Moreover, our finding was agreed with the results of previous studies done by ${ }^{23-}$ ${ }^{25}$ that have found that a dominance of Gram-negative bacteria that may be related to the colonization of the hands of healthcare providers. Similarly, our findings are supported by similar study done by ${ }^{26}$ who showed a predominance of Gram-negative bacteria causing BSI in a Pediatric ICU. On the other hand, our finding is contradicted with a similar study done by ${ }^{27}$ who found gram positive cocci were the most common organisms colonizing CVCs followed by Gram negative Bacteria.

Regarding sensitivity of bacteria to antibiotics, the present results revealed $S$. aureus and $S$. epidermidis that isolated from nosocomial BSI cases were sensitive to Vancomycin, Amikacin and levofloxacin. This finding is partially in line with similar study

done by Khanna et $\mathrm{al}^{28}$ who reported that all resistant Staphylococcus isolated from CRLI were 100\% sensitive to vancomycin, teicoplanin, and linezolid . Moreover, our finding is congruent with the results of the study done by Curtis et $\mathrm{al}^{29}$ who conducted a study about catheter-related bloodstream infection in the intensive care unit and found all isolates were sensitive to Vancomycin and Teicoplanin. Concerning studying the antibiotic resis- tance of $S$. epidermidis isolates and $S$. aureus in the current study, it revealed that the majority of cases were resistent to amoxycillin and meropenem, on the other hand, half of cases were resistent to olfloxacine, clindamycin and ceftriaxone

Potential risk factors for BSI have been mentioned in previous studies, e.g., type of catheter, duration of catheterization, site of insertion, length of hospital stay, triple lumen catheter, and duration of catheter in place. In our study, we observed an overall average of hospital stay was ten days and all the cases stayed more ten have been associated with blood stream infection. This finding is supported by Mitt et $\mathrm{al}^{23}$ who studied Epidemiology of nosocomial bloodstream infections in Estonia and reported that additional days of hospital stay have been associated with higher BSI rates

Whether the number of lumens in the CVCs was associated with a higher rate of infection is controversial. In our study, the nurses utilized triple lumen for all engaged patient. The possible explanation for selecting triple lumen was administration of parentral fluids, medications, blood products and measuring CVP. Therefore, the catheter was manipulated many times per day. Our finding is in agreement with ${ }^{30-31}$ who detected in the multivariate analysis that the possibility of acquiring a bloodstream infection was high when a patient had used a multiple-lumen CVC compared to a one -lumen catheter

Regarding the setting of patients' insertion of central venous catheters, the present findings revealed that all cases were inserted in the ICU setting and nearly half of them were due to emergency causes. Accordingly, the incidence of infection was higher. The possible explanation is giving greater attention to aseptic technique during the time of insertion in emergency cases might not be applicable and the catheters can be handled many times daily for hemodynamic measurements or to obtain samples. Besides, the dominance of nosocomial infections in ICU. Our results are in line with ${ }^{32}$ who reported that the occurrence of infecton is higher in ICU settings than in the less acute settings. They have attributed the possible factors to central venous entrance might be needed for long periods of time; patients can be colonized with intensive care-acquired organisms; and the catheter can be handled many times daily for administering fluids, medications, and blood transfusion. 
It has been proven that the site of central venous catheter insertion may be a significant risk factor for the induction of blood stream infection ${ }^{25}$. The catheters inserted in the jugular vein are more prone to get infection than those inserted in the sub-clavian vein. This may be related to factors that promote contamination of the skin next to jugular vein such as spread of sputum dropplets, difficulties in catheter fixation and frequent dressing ${ }^{17}$.

As well, Parienti et $\mathrm{al}^{33}$ studied a meta-analysis of central venous catheter-associated Infection risk reduction in critically ill patients and revealed that the sub-clavian vein site was associated with lower catheter-related infections compared with other sites. In our study, nearly two third of cases underwent insertion of $\mathrm{CVC}$ through jugular route. However, the incidence of blood stream infection was higher in patients with sub-clavian route when compared to jugular route. The possible rationale for this finding may be related to the insertion technique of sub-clavian venous catheter is not being easy and requires more time and trials during insertion. As well, insertion of CVC catheter utilizing sub-clavian route consumed more than 20 minutes during insertion utilizing guide wires.

Referring to the technique of Central venous catheter insertion is often difficult and embarrassing in obese patients leading to masked landmarks of the neck. ${ }^{34}$ In addition, excessive sweating, dressing at the site of CVC insertion are frequently accompanied by blood stream infection. ${ }^{16}$.

In the present study, more than half of blood stream infection cases were overweight and obese. Our finding is contradicted with ${ }^{35}$ who Compared the CRBSI rate in obese and in non-obese and found no differences in CRBSI . In our study, the CRBSI rate in patients with diabetes mellitus was higher when compared to overall population. The authors' point of view that those diabetic patients may have low immunity and the CVC site of insertion is good port of entrance of microorganisms. These findings are consistent with that of ${ }^{36}$ who conducted a study about clinical outcomes in critically ill patients with bloodstream infections and found the proportions of diabetes mellitus were greater among patients with BSI compared to patients without BSI

\section{Conclusion}

The study concluded that out of 120 patients in the pres- ent study, 13 patients acquired bacterial blood stream infection and revealed positive blood culture 7 days after insertion of central venous catheters. Out of thirteen infected cases, 10 cases were infected with Staphylococcus epidermis.

\section{Limitations}

Our study was limited to generalize the current findings due to small sample size confined to one hospital .Therefore, our study recommended replication of the study on a larger probability sample from different geographical locations.

\section{Conflicts of interest}

None declared.

\section{Acknowledgments}

We express our sincere appreciation to all critical care nursing staff and infection control staff at $\mathrm{Al}$ Haram hospital for their cooperation.

\section{References}

1-Ren H, Colletta A, Koley D, et al. Thromboresistant/ anti-biofilm catheters via electrochemically modulated nitric oxide release. Bio Electro Chem 2015;104:10-16. 2-Lona-Reyes JC, López-Barragán B, De La Rosa AJ, et al.. Central venous-catheter related bacteremia: incidence and risk factors in a hospital in western Mexico. Boletín Médico del Hospital Infantil de México (English ed.). 2016;73(2):105-110.

3-Shah H, Bosch W, Thompson KM, et al.. Intravascular catheter-related bloodstream infection. The Neuro Hospitalist. 2013;3(3):144-151.

4-Hammarskjöld F, Berg S, Hanberger H, et al.. Sustained low incidence of central venous catheter-related infections over six years in a Swedish hospital with an active central venous catheter team. Am J Infect Control. 2014;42:122-128.

5-Ullman, AJ, Long, DA, Rickard, CM (2014). Prevention of cen-tral venous catheter infections: A survey of paediatric ICU nurses' knowledge and practice. Nurse Education Today 34, 202-207. https://doi.org/10.1016/j. nedt.2013.09.002.

6-Eggimann P, Pittet D. Overview of catheter related infections with special emphasis on prevention based on educational programs. Clin Microbiol Infect 2002;8:295-309. 7-Donlan RM. Biofilms and device associated infections. Emerg Infect Dis 2001;7:277-81. 
8-El-Kholy A, Tamer S, Gaber M, et al. (2012). Device-associated nosocomial infection rates in intensive care units at Cairo University hospitals: First step toward initiating surveillance programs in a resource-limited country, Egypt, American Journal of Infection Control; 40 e216- 20.

9-Koneman EW, Allen SD, Janda WM, Schreckenberger PC, Winn WC Jr. Charts. In: Darcy P, Pterson N, Montalbano J, editors. Koneman's Color Atlas and Textbook of Diagnostic Microbiology. 6th edition. Philadelphia: Lippincott; 1997. p. 1442-1535.

10-Cheesbrough M.(2006). District Laboratory Practice in Tropical Countries. $2^{\text {nd }}$ ed., Cambridge University Press: pp. 100-194.

11-Clinical and Laboratory Standards Institute. Performance Standards for Antimicrobial Susceptibility Testing. Twenty-Fourth Informational Supplement. M100-S24, Vol 34, No 1: Wayne, Pennsylvania; 2014. p. 38-42.

12-Yaghmaie F. (2009). Content validity and its estimation. Journal of Medical Education; 3: 25-27.

13-Polit DF, Beck CT, Owen SV. Is the CVI an acceptable indicator of content validity? Appraisal and recommendations. Res Nurs Health. 30, 459-467 (2007).

14-Lawshe CH. A quantitative approach to content validity. Personnel Psychol. 28, 563-575 (1975).

15-Culshaw N, Glover G, Whiteley C, Rowland K, Wyncoll D, Jones A, Shankar-Hari M.(2014). Healthcare-associated bloodstream infections in critically ill patients: descriptive cross-sectional database study evaluating concordance with clinical site isolates, Annals of Intensive Care. http://www.annalsofintensivecare.com/content $/ 4 / 1 / 34$

16-O'Grady NP, Alexander M, Dellinger EP et al. (2002). Healthcare Infection Control Practices Advisory Committee. Guidelines for the prevention of intravascular catheter-related infections. Centers for Disease Control and Prevention. MMWR Recomm Resp; 51:1-29.

17-Trick WE, Miranda J, Evans AT, Charles-Damte M, Reilly B, \& Clarke P.(2006). Prospective cohort study of central venous catheters among internal medicine ward patients. Am J Infect Control; 34:636-641.

18-Tacconelli E, Smith G, Hieke K et al. (2009). Epidemiology, medical outcomes and costs of catheter-related bloodstream infections in intensive care units of four European countries: literature and registry-based estimates. $J$ Hosp Infect, 72:97-103.

19- Rosenthal VD, Maki DG, Mehta A et al. (2008). The International Nosocomial Infection Control Consortium
(INICC): Goals and objectives, description of surveillance methods, and operational activities. Am J Control; 36(9):627-37.

20-Rosenthal VD, Maki DG, Salomao R et al.(2006). International Nosocomial Infection Control Consortium. Device-associated nosocomial infections in 55 intensive care units of 8 developing countries. Ann Intern Med, 145:582-91.

21 - Hussain N, F Salama, M Yehia, N Haifaa, H Al-Mousa AH, Alhilali N.(2010) Infection related processes during haemodialsis: A study in General Hospital Haemodialysis unit, Egypt

22- Singh KM, Mallan D, Tripathi SS. Yadav RR, Avasthi S. (2016).A study of central Venous Catheter Colonizations and Bood stream Infections among patients admitted in the Intensive Care Unit of a Tertiary Care Teaching Hospital .

23- Mitt P, Adamson V, Loivukene K, Lang K, Telling K, Paro K, Room A, Naaber P, Maimets M.(2009). Epidemiology of nosocoomial bloodstream infectins in Estonia. J Hosp Infect. Apr;71(4):365-70. doi: 10.1016/j. jhin.2009.01.008.

24- Van der Kooi TI, Boer AS, Mannien J et al. (2007). Incidence and risk factors of device associated infections and associated mortality at the intensive care in the Dutch surveillance system. Intensive Care Med; 33(2):271-8.

25-Lorente L1, Henry C, Martin MM, Jimenez A, Mora ML.(2005). Central venous catheter-related infection in a prospective and observational study of 2,595 catheters. Crit Care;9(6):R631-5.

26- Abramczyk ML, Carvalho WB, Carvalho ES et al. (2003). Nosocomial infection in a pediatric intensive care unit in a developing country. Braz J Infect Dis; 7(6):375-80. 27-Kaur M, Cupta V, Gomhar S, Chander J, Ahoo $\mathrm{T}(2015)$. Incidence ,risk factors , microbiology of venous catheters associated blood stream infections - a prospective study from a tertiary care hospital. Indian J Med Microbiol; Apr-June ; 33(2):248- 254.

28- Khanna V, Mukhopadhayay C, Vandana KE, Verma M, Dabke P.(2013). Evaluation of Central Venous Catheter Associated Blood Stream Infections: A Microbiological Observational Study. Journal of Pathogens., Article ID 936864, 6 pages. http://dx.doi. org/10.1155/2013/936864.

29- Curtis R.L.(2009). Catheter-related bloodstream infection in the intensive care unit. The Intensive Care Society Journal, April .; volume 10, Number 2. 
30-Moretti EW, Ofstead CL, Kristy RM et al. (2005) Impact of central venous catheter type and methods on catheter-related colonization an bacteremia. J Hosp Infection; 61:139-45.

31- Templeton A, Schlegel M, Fleisch F et al. (2008). Multilumen central venous catheters increase risk for catheter-related bloodstream infection: prospective surveillance study. Infection; 36:322-27.

32-O'Grady NP, Alexander M, Burns LA, Dellinger EP, Garland J, Heard SO et al.(2011). Guidelines for the prevention of intravascular catheter-related infections. Clin Infect Dis. , 52: e162-e193. 10.1093/cid/cir257.

33- Parienti JJ, du Cheyron D, Timsit JF et al.(2012). Meta-analysis of subclavian insertion and non-tunneled cen- tral venous catheter-associated infection risk reduction in critically ill adults. Crit Care Med.; 40:1627e1634.

34- Graham AS, Ozment C, Tegtmeyer K, Lai S, Braner DA(2007). Central venous catheterization. $N$ Engl J Med; 356:e21.

35-Schalk E, Farber J, Fischer T, Heidel HF. (2015). Central Venous Catheter- Related Bloodstream Infections in Obese Hematologic Patients. Infection Control \& Hospital. Epidemiology; august; vol. 36, no. 8.

36-Apostolopoulou E, Zikos D, Tselebis A, Drosatou X, Stefanidis E, Xristodoulou A, Katsaris G.(2010). Clinical outcomes and economic variables in critically ill patients with bloodstream infections. Health Science Journal. Volume 8 (2014), Issue 4. 